\title{
Context-Awareness based Driving Assistance System for Autonomous Vehicles*
}

\author{
Minwoo Ryu ${ }^{1}$ and Si-Ho Cha ${ }^{2 *}$ \\ ${ }^{1}$ Service Laboratory Institute of Convergence Technology, \\ KT R\&D Center, 151 Taebong-ro, Seocho-gu, 06763, South Korea \\ ${ }^{2}$ Dept. of Multimedia Science, Chungwoon University \\ 113, Sukgol-ro, Nam-gu, Incheon, 402-803, South Korea \\ ${ }^{1}$ mw.ryu@kt.com, ${ }^{2}$ shcha@chungwoon.ac.kr
}

\begin{abstract}
Through numerous artificial intelligence (AI) technologies advances, a new type of system, so-called advanced driving assistance system (ADAS) have been introduced and interested in vehicle driving space. The ADAS can assistance driving for driver using different entities including lane-keeping assist, adaptive cruise control and pre-crash system. Recently, many researchers have been proposed self-driving system using the ADAS. In this sense, we proposed a design and implementation of driving assistance system for autonomous based on context-awareness of surrounding environment of vehicles. To this end, we design and implement an ontological model of context information within vehicle space. In addition, we develop a testbed using smart video car kit in our testbed.
\end{abstract}

Keywords: Self-Driving Car, Ontology, Context-Awareness, ADAS, AI

\section{Introduction}

Through numerous artificial intelligence (AI) technologies advances, a new type of system, so-called advanced driving assistance system (ADAS) have been introduced and interested in vehicle driving space [1]. The ADAS is used to assistance driving for driver using advanced functions such as lane-keeping assist (LKA), adaptive cruise control (ACC) and pre-crash system PCS) [2]. Accordingly, the ADAS is one of key technologies to realize autonomous vehicle era. Due to these reasons, the ADAS have been became main research topic in past 10 years. For example, the European New Car Assessment Programme (EuroNCAP) announced its introduction of automated emergency braking system (AEBS) test from 2014 [3]. And also, DARPA challenge started from 2004 accelerated the research for autonomous driving [4]. In addition, Several OEMs and universities have been demonstrated self-driving car and developed their prototypes [5].

A conventional ADAS is composed of sensing, perception, planning and operation. Here, the sensing is used to collect information about driving environment in/out vehicle using sensor such as LiDAR, and camera. The perception is used to analyze and understand current driving environment using diverse intelligence algorithms. The planning is used to define timing based on analyzed results using pre-stages (i.e., sensing and perception) information. Finally, operation is used to control vehicle [5-8].

\footnotetext{
* This paper is a revised and expanded version of a paper entitled 'Design of Driving Assistance System for Autonomous Vehicle based on Context-Awareness' presented at GST2017, Jeju National University, Jeju, Korea, 1st December, 2017.

Received (October 17, 2017), Review Result (December 19, 2017), Accepted (January 20, 2018)

* Corresponding Author: Si-Ho Cha
} 
Recently, through acceleration of autonomous vehicle and high autonomous vehicle realized by $5 \mathrm{G}$ (5-generation) and vehicle to everything (V2X) communication, comprehensive situational awareness is required in the current ADAS. However, unmarked roads, and unknown driving situation could not be fulfilled in the conventional ADAS. To resolve this problem, we proposed a novel context-awareness based driving assistance system for autonomous vehicle. To this end, we design an ontological model could be called vehicle context ontology for vehicle space. And then, we develop the ontology model using protégé [10]. Finally, to show the feasibility of the proposed context ontology, we develop a testbed using the smart video car kit which is composed of raspberry-pi and divers sensors and line tracer.

The rest of this paper is organized as follows. The existing related work in this research area is introduced in Section 2. The detail of the vehicle context ontology is described in Section 3. Section 4 presents a testbed to evaluate the proposed context ontology. Finally, we conclude our remarks in Section 5.

\section{Related Works}

In driving vehicle space, a context-awareness include comprehensive situation such as road environment, and driving environment of in/out-vehicle. According to autonomous vehicle and high autonomous vehicle, many research work have been proposed to improve the effectiveness of the ADAS.

Chunzhao et al. proposed a stereovision-based multimodal ADAS [10]. In this article, the proposed system expands the usability of conventional ADAS functions in unmarked road. In particular, the system improves low-level detection of object of interest than conventional ADAS and estimate high-level road structure in probabilistic reasoning procedures. Accordingly, the system can help increase the value of the existing ADAS without major modifications or expense.

Alexandre et al. proposed an ontology-based context awareness [1]. In this article, the authors propose a taxonomy of ontology defined by 3 major classes including the Mobile entities, Static entities, and Context parameters in vehicle area. In addition, they show how ontologies can be used as a tool in ADAS via 1-D scenario-based experiment.

Yiwen et al. proposed a framework named CASD (Context-Awareness Safety Driving) [11]. The proposed framework enables to provide safety action plan by three situations including the Line-of-Sight unsafe, Non-Line-of-Sight unsafe and safe situation. To this end, the authors define new service layer could be called context-awareness safety driving upper LLC layer in WAVE framework. Accordingly, the CASD can run on GPS navigator and DSRC devices. In addition, the CASD classifies neighboring vehicles into 3 main classes including mobility information, sensors information, and driver's behaviors. Through this classification, the CASD enables to provide different action plans for driving safety for each class.

Amirton et al. proposed a context-awareness system to provide drivers without distracting from driving activities in smart cities [12]. To this end, they defined context information model will be the basis to the ontology for vehicular application. In particular, the model focused on the quality of information (QoI) to drivers and autonomous vehicles.

Alexandre et al. proposed principle of a framework which allows the estimation of the degree of awareness in vehicular behavior [13]. In this article, the proposed framework can learn the manner about individual driver's behavior via given context. After learning, the framework can detect whether or not the driver is behaving differently under similar conditions. Accordingly, the framework enables to estimate driver awareness with particular contextual object.

Recently, many research work have been trying to aware context in/out vehicle in order to achieve requirements of autonomous vehicle or high autonomous vehicle. 
However, most proposed technologies predefined several scenarios in order to aware context of in/out vehicle. In order to achieve requirements of autonomous vehicle and high autonomous vehicle, we have to dynamically aware context of in/out vehicle. In this perspective, we proposed novel context awareness model based ontology.

\section{Context Awareness based Assistant Driving System}

In this section, we describe propose context awareness based assistant driving system. The proposed system enables to provide divers services including context awareness service, and interoperable vehicle service with internet of things (IoT). Figure 1 shows concept of service range based on the proposed system.

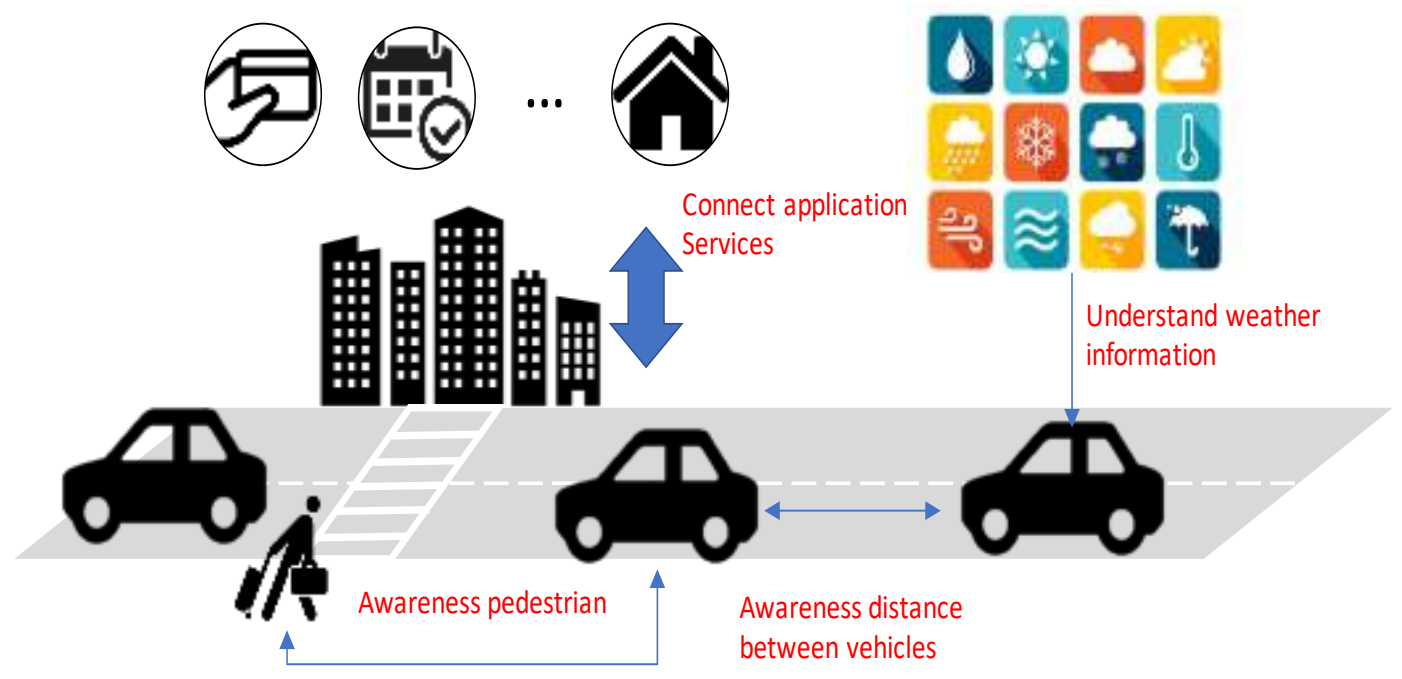

Figure 1. Concept of Service Range of the Proposed System

To this end, the proposed system is divided into three main parts. First part is design of vehicle context ontology. In this part, we describe an ontological model for vehicle space. Second part is design of framework for the system. In this part, we introduce architecture and functions of proposed system. Finally, we explain development of system including ontology.

\subsection{Design Vehicle Context Ontology}

In this section, we describe design of vehicle context ontology. The proposed context ontology enables to understand and interpret meaning of data from in/out sensors and device, and then to aware context of current driving environment. In addition, the ontology allows to provide interoperable services with Internet of Things (IoT).

The vehicle context ontology is composed of 4 ontologies including the oneM2M Base Ontology [14], Semantic Sensor Network Ontology [15], and a vehicle taxonomy ontology (VO).

The oneM2M Base Ontology is designed by WG 5 (i.e., MAS) in oneM2M standard. The ontology allows to describe IoT services and IoT entities such as devices, data types, and functions. The oneM2M standard is one of standard development organization for IoT.

In vehicle context ontology, the oneM2M Base Ontology is used to represent IoT services interworked with vehicle services. In particular, this ontology is used to provide dynamic IoT services according to results of context awareness in driving environment. 
The SSN designed by semantic sensor network incubator group in W3C. The ontology can describe diverse sensors, observations, and related concept. In the vehicle context ontology, the SSN is used to represent vehicle in/out sensors such as LiDAR, and camera as well as environment sensors such as temperature sensor and humidity sensor located in road side.

Finally, the VO is used to represent vehicle information. In particular, this ontology includes information of current weather and road type. Figure 2 shows proposed vehicle context ontology.

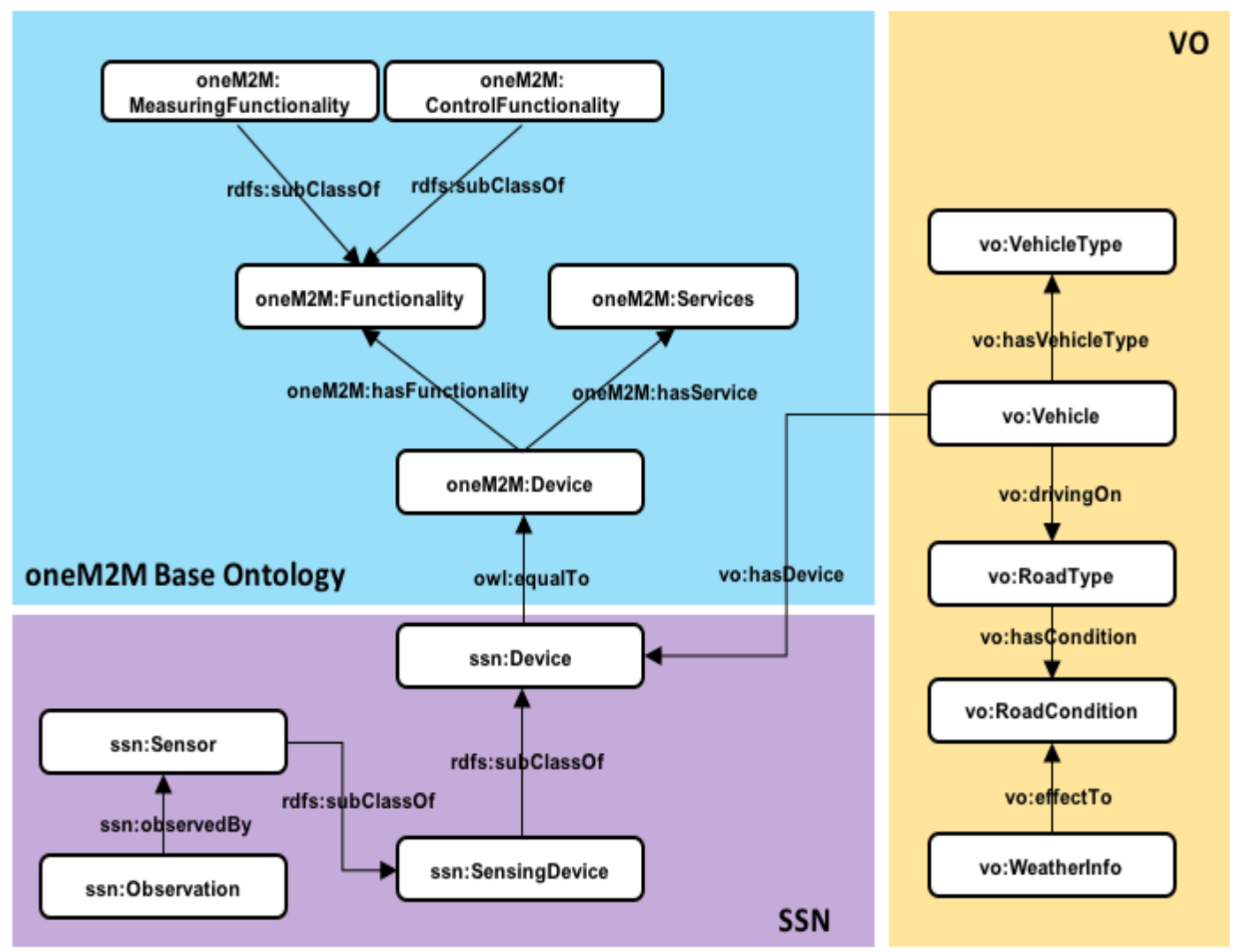

Figure 2. Vehicle Context Ontology

In Figure 2, rectangle describes classes and arrow describe object properties. And also, The VO is highlighted in yellow color and SSN is highlighted in purple. Finally, the oneM2M Base Ontology is highlighted in blue.

In the VO, the Vehicle class represent a vehicle entity. Accordingly, name of vehicle is defined as an individual of this class. The Vehicle Type class represent kind of vehicle such as bus, and truck, and automobile. The RoadType class represent road type such as intersection, straight, urban, and highway. The RoadCondition class represent condition or road. The WeatherIfo class represent weather information. The Vehicle class has relation with VehicleType class and RoadType class via object properties named hasVehicleType and drivingOn, respectively.

The RoadType class has relation with RoadCondition through hasCondition. Finally, the RoadCondition class has relation with WeatherInfo using effectTo.

In the oneM2M Base Ontology, the Device class represent a device located in vehicle or out vehicle. This class has relation with SSN and VO using this class. The Functionality class represent functions of a device. And this class has sub-class named MeasuringFunctionality and ControlFunctionlity, respectively. The Service class 
represent service of a device. The Device class has relation with Functionality class and Service class via hasFunctionality and hasService, respectively.

In SSN, SensingDevice class is a sub-class of the Device class, and represent sensor information. The sensor class is a sub-class of the SensingDevice. In this class, each individual is a specific kind of sensor. The Observation class represent functions of sensors, in particular measuring function.

Each ontology has relationship with object properties between each other. In other words, Vehicle class of VO has relation with the Device class of SSN through object property named hasDevice. And, the Device class of SSN has same meaning with Device class of oneM2M Base Ontology.

\subsection{Architecture of Driving Assistant System}

In this section, we present architecture of driving assistant system. The system is divided into 2 main parts. First is a software which run on vehicle side. Another one is a server side. Figure 3 shows architecture of the system.

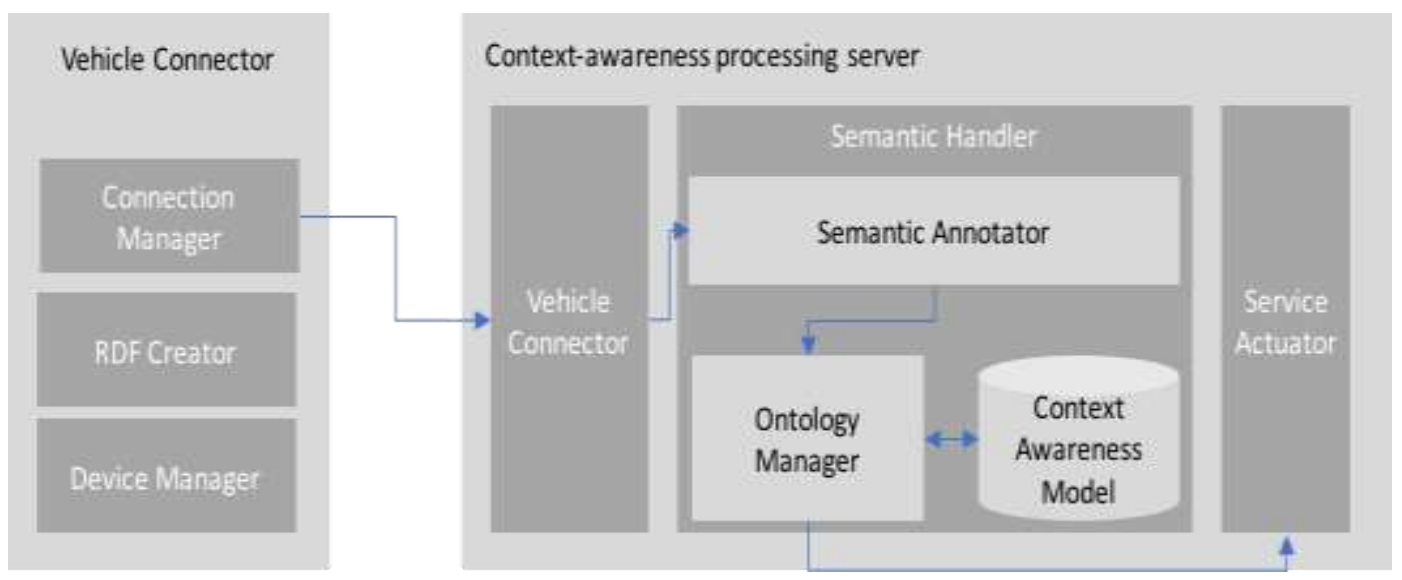

Figure 3. Architecture of Driving Assistant System

As shown Figure 3, the system is composed of 2 main packages which is the Vehicle Connector and Context-awareness processing server. The Vehicle Connector is a client software which run on vehicle inside.

The Vehicle Connector is composed of 3 software components including the Device Manager, RDF Creator, and Connection Manager.

The Device Manager manages sensors and devices are equipped in vehicle. To do this end, the Device Manager has configuration file to define connected device. According, sensors equipped in vehicle are defined in the configuration file. This configuration file is used to sharing sensing information with the Context-awareness processing server. Another work of the Device Manager, collect information from sensors and then send the information to the RDF Create component. This information is going to create RDFs in the RDF Creator component.

The RDF Creator performs create RDF in order to upload to the Context-awareness processing server. The RDF Creator receive sensing information from the Device Manager component, and then the RDF Creator create RDFs. Here, SSN is base ontology in order to create RDF. After creation of RDF, this RDF is uploaded to the Connectawareness processing server via the Connection Manager component.

The Context-awareness process server consists of 3 components such as the Vehicle Connector, Semantic Handler, and Service Actuator.

The Vehicle Connector is interface in order to connect with vehicles. In particular, this interface is used to receive RDF data from the Vehicle Connector. 
The Semantic Handler performs handling of RDF data. To this end, the Semantic Handler is composed of 3 software blocks including the Semantic Annotator, Ontology Manager, and Context Awareness Model.

The semantic Annotator performs a function which combine RDF data and context model. In other words, RDF data received from vehicle side, are became an individual of vehicle context ontology. According, annotated data are stored in the Context Awareness Model through the Ontology Manager component.

The Ontology Manager performs management of vehicle context ontology. This component has functions which are CRUD (Create, Retrieve, Update, and Delete) about the ontology.

The Context Awareness Model is a database for ontology, and vehicle context ontology is stored in the database.

Finally, the Service Actuator performs service triggering in order to provide service to driver such as safety service, and interworking service with IoT.

\subsection{Implementation}

In this section, we develop vehicle context ontology and system. To develop vehicle context ontology, we use protégé. Figure 4 shows implementation of vehicle context ontology.

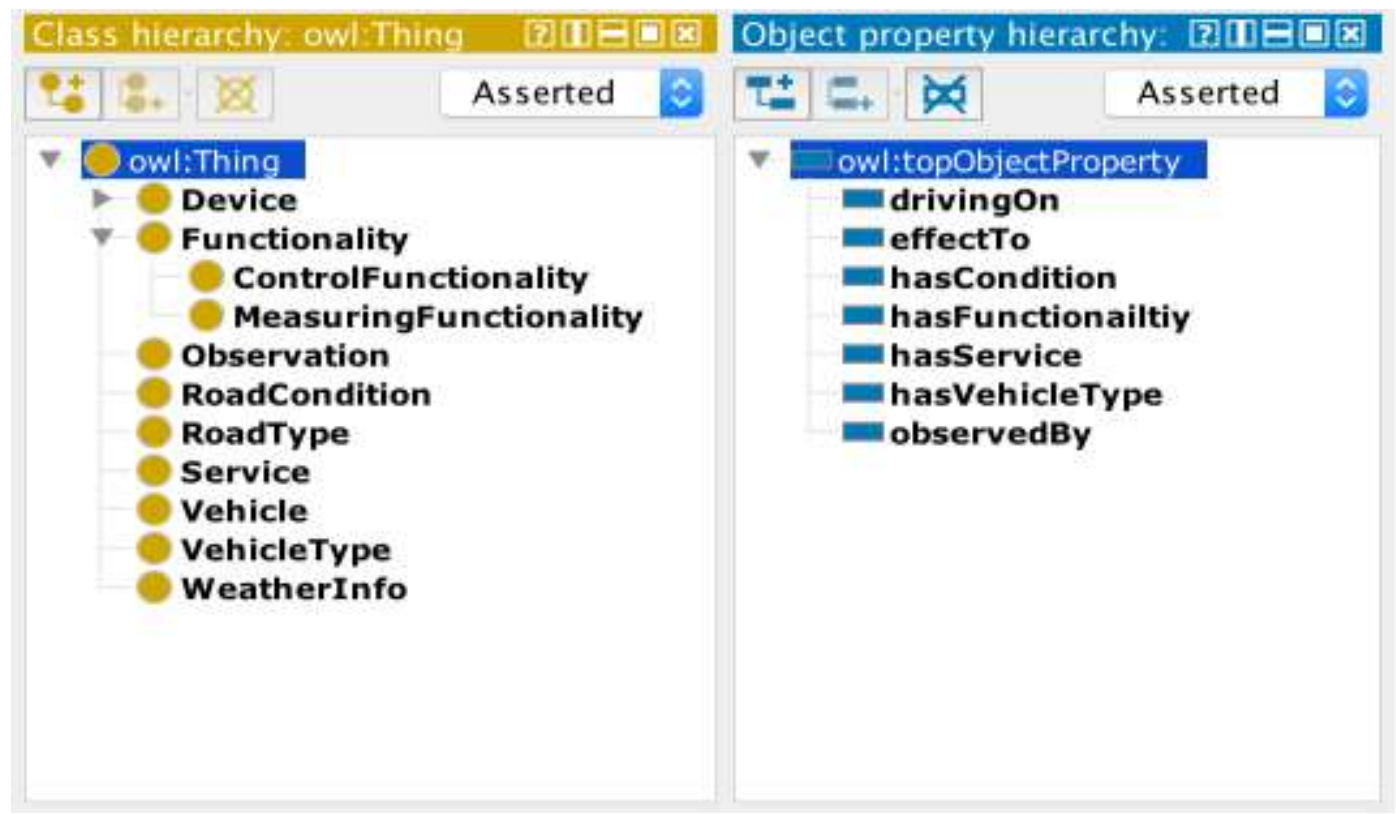

\section{Figure 4. Implementation of Vehicle Context Ontology Using Protégé}

In Figure 4, the yellow circles denote classes of the ontology, and blue rectangles denote object properties.

The Driving assistant system is developed using Java, the Jena library, the SPARQL, and Jena TDB (Turtle DB). For the vehicle side, the Vehicle Connector is developed by Java, and then is equipped in raspberry-pi.

For the Context awareness processing server, we developed the web application server (WAS), i.e., Tomcat 8.0. In the server, we developed the spring frame work in order to provide interface to the Vehicle Connector. We also developed the Semantic Annotator and Ontology manager modules using the Jena library. In addition, we developed semantic queries for ontology management using the SPARQL. Finally, we developed ontology database using Jena TDB. 


\section{Performance Evaluation}

In this section, we evaluate the performance of the proposed system. To do this end, we developed the testbed using the Smart video car kit [16] and vehicle running track.

To sense driving environment, we add diverse sensors including camera, ultrasonic sensor, temperature sensor, and humidity sensor. Camera sensor is used to monitor road type and monitor surrounding environment of vehicle. Temperature sensor and humidity sensor are used to check in/out side temperature and humidity of vehicle. Finally, the Vehicle Connect package is installed in raspberry-pi of the smart video car kit. Figure 5 shows the Smart video car kit.

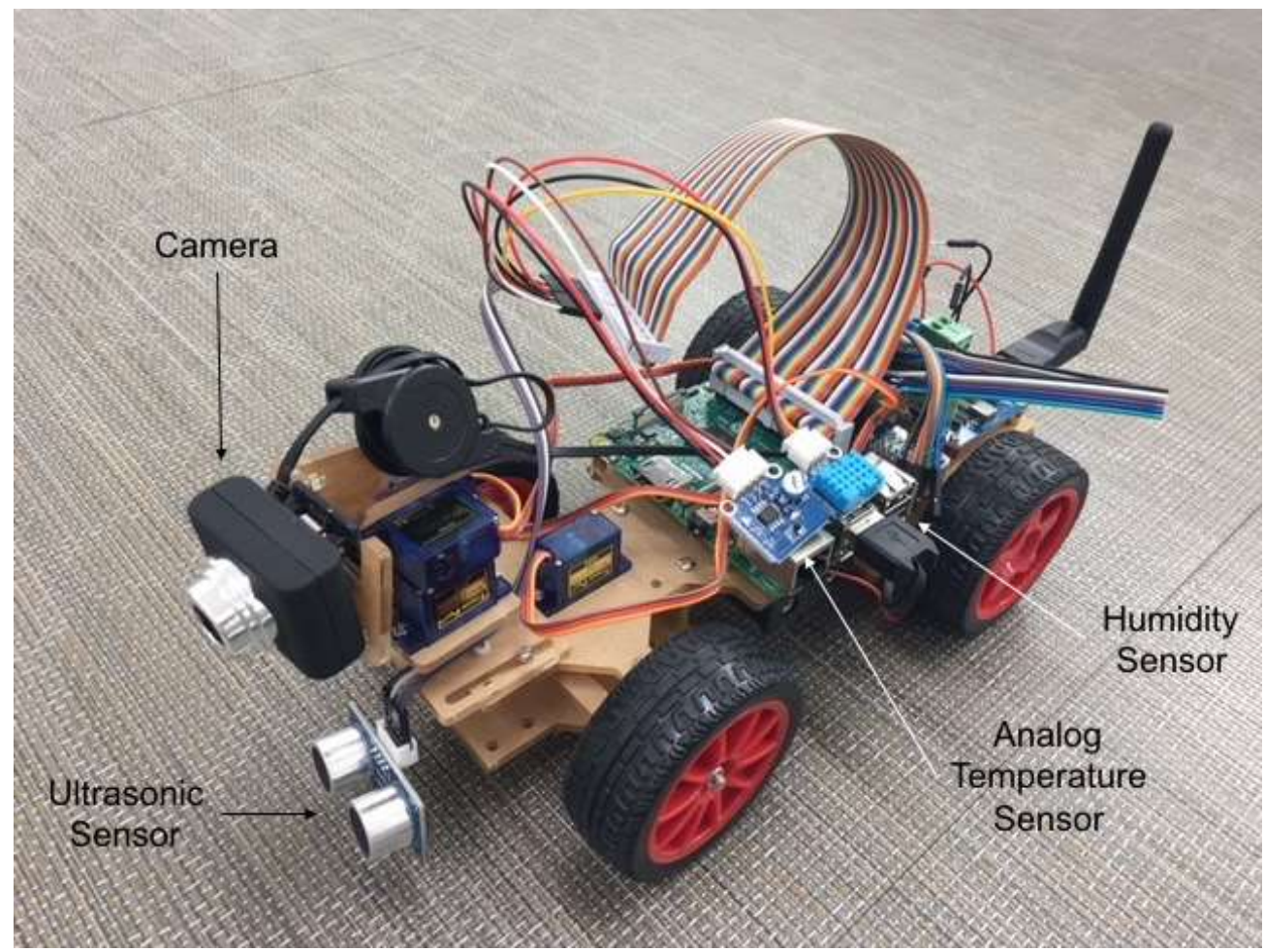

Figure 5. Smart Video Car Kit

To evaluate the proposed context model and system, we build line tracer. And then, we test the model and system with smart video car kit. To this end, we define two scenarios including self-driving scenario, and weather scenario. In self-driving scenario, the smart video kit is drove by itself.

In the weather scenario, the smart video kit is drove by itself as self-driving scenario. But, the smart video car kit controls its velocity according to road condition. For this, we made rainy and shiny. Figure 6 shows a snapshot of first scenarios (i.e., self- driving scenario) 


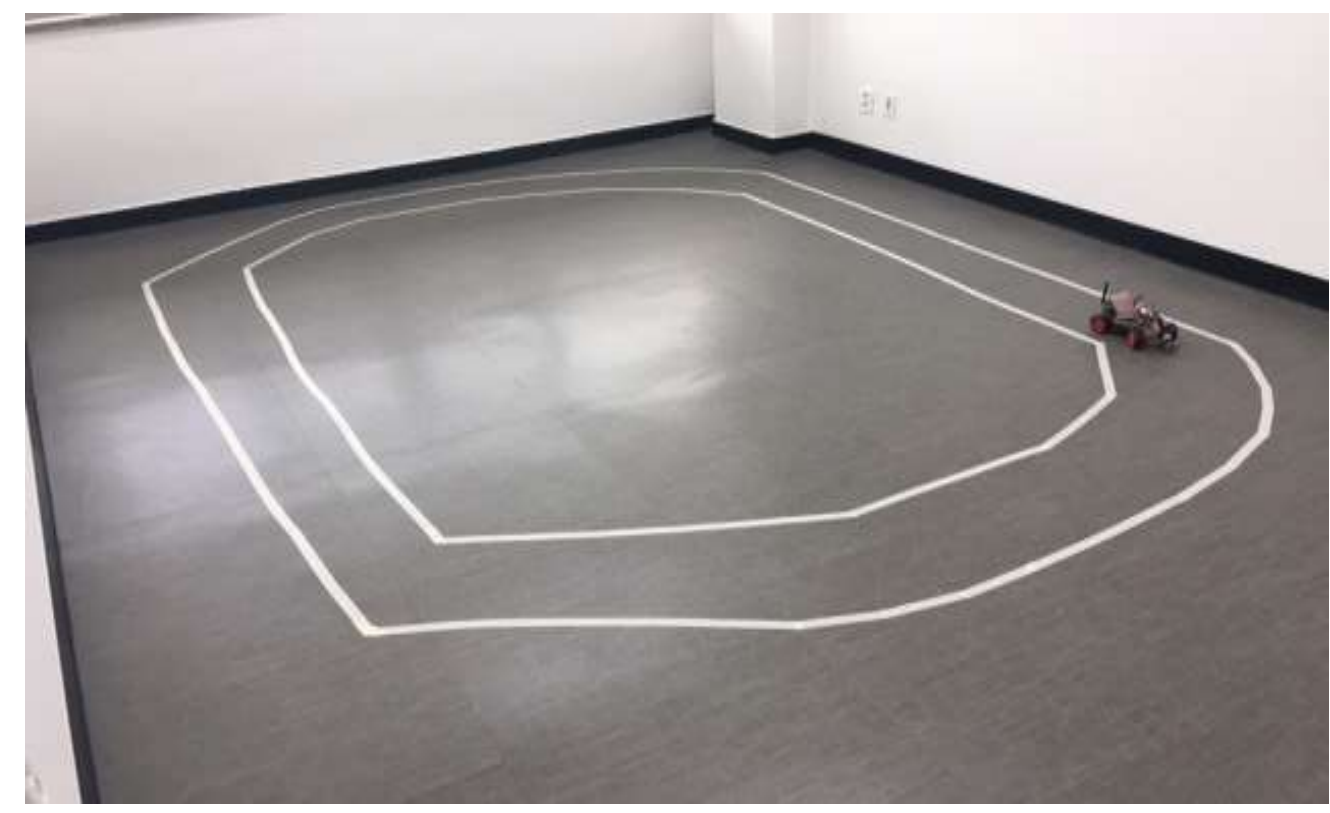

Figure 6. Snapshot of Self-Driving

\section{Conclusion}

In this paper, we proposed design and implementation of driving assistance system for autonomous vehicle and high autonomous vehicle based on context awareness. To this end, we design and implementation of vehicle context ontology. In particular, the proposed context ontology allows to easily combine between different ontologies because the proposed ontology include standard-based ontologies, i.e., SSN and oneM2M Base Ontology. In addition, we developed the driving assistant system which is composed of the Vehicle Connector and Context awareness processing server. In the system, through separation between vehicle side and server side, we enable to provide faster context awareness system.

Finally, we show feasibility the proposed system using real testbed. For the evaluation, we developed vehicle using the smart video car kit and vehicle running track for evaluation. In addition, we defined two scenarios including self-driving and weather information-based self-driving in order to test various driving environment. Consequently, we can conclude with confidence that the proposed system can eventually contribute to realize autonomous vehicle and high autonomous vehicle.

However, the proposed system is tested in prototype level. In order to show feasibility, we need test in field. In addition, to provide more intelligence services to driver, we need interoperable service between different domains. This is a challenge task to realize autonomous vehicle and high autonomous vehicle.

\section{Acknowledgments}

This research was supported by Basic Science Research Program through the National Research Foundation of Korea (NRF) funded by the Ministry of Education (NRF2016R1D1A1A09917662).

\section{References}

[1] A. Armand, D. Filliat and J. Ibañez-Guzman, "Ontology-based context awareness for driving assistance systems”, 2014 IEEE Proceedings on Intelligent Vehicles Symposium, IEEE, (2014), pp. 1-7.

[2] Advanced Driver Assistance System. Available online: http://en.wikipedia.org/wiki/Advanced_driver_assistance_system (accessed on18 January 2018).

[3] Euro NCAP, Available online: https://www.euroncap.com/en (accessed on 18 January 2018). 
[4] The DARPA Grand Challenge: Ten Years Later, Available online: https://www.darpa.mil/newsevents/2014-03-13 (accessed on 18 January 2018).

[5] R. Okuda, Y. Kajiwara and K. Terashima, "A survey of technical trend of ADAS and autonomous driving", Proceedings of Technical Program-2014 International Symposium on VLSI Technology, Systems and Application (VLSI-TSA), IEEE, (2014), pp 1-4.

[6] K. A. Brookhuis, D. De Waard and W.H. Janssen, "Behavioural impacts of advanced driver assistance systems-an overview", European Journal of Transport and Infrastructure Research, vol. 1, no. 3, (2001), pp. 245-253.

[7] Christopher P. Ricci, "Customization of vehicle user interfaces based on user intelligence", U.S. Patent Application, No. 14/253, (2014).

[8] D. Schnabel, J. Wang, J. Straub and E. Kim, "Artificial Intelligence in an Automated Vehicle Starter", University of North Dakota Graduate School Scholarly Forum, (2016).

[9] Protégé for ontology editor. Available online: https://protege.stanford.edu (accessed on 18 January 2018).

[10] C. Guo, J. Meguro, Y. Kojima and T. Naito, "A multimodal ADAS system for unmarked urban scenarios based on road context understanding", IEEE Transactions on Intelligent Transportation Systems, vol. 16, no. 4, (2015), pp. 1690-1704.

[11] Y. Shen, J. Jeong, T. Oh and S. H. Son, "CASD: a framework of context-awareness safety driving in vehicular networks", 2016 30th International Conference on Advanced Information Networking and Applications Workshops (WAINA), IEEE, (2016), pp. 252-257.

[12] A. B. Chagas and C. A.G. Ferraz, "Smart Vehicles for Smarter Cities: Context-Aware V2X to Improve QoI", Anais do XXIII Simpósio Brasileiro de Sistemas Multimídia e Web: Workshops e Pôsteres, (2017), pp. 64-69.

[13] A. Armand, D. Filliat and J. Ibanez-Guzman, "Detection of unusual behaviours for estimation of context awareness at road intersections", 5th Workshop on Planning, Perception and Navigation for Intelligent Vehicles, (2013), pp. 313-318.

[14] oneM2M BaseOntology. Available online: http://www.onem2m.org/technical/ developerscorner/tools/onem2m-ontologies (accessed on 18 January 2018).

[15] SSN (Semantic Sensor Network) Ontology. Available online: semantic sensor network ontology download (accessed on 18 January 2018).

[16] Smart Video Car Kit, Available online: https://github.com/sunfounder/Sunfounder_Smart_Video_Car_Kit_for_RaspberryPi (accessed on 18 January 2018).

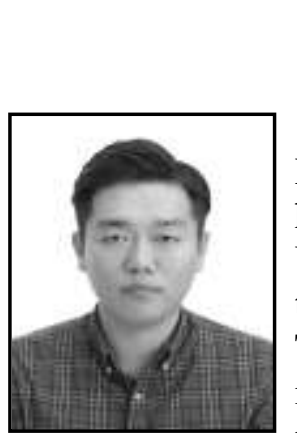

\section{Authors}

Minwoo Ryu, he received his B.S degree in Internet Information Processing from Yejou Institute of Technology, Korea in 2007, and his M.S and Ph.D. degrees in Computer Science from Kwangwoon University, Seoul, Korea in 2009 and 2012, respectively. From 2011 to 2016, he worked as a research scientist at Korea Electronics Technology Insititute (KETI), Korea. Ho is now a research scientist in the KT R\&D center, Korea Telecom (KT), Korea. His research interests include Internet of Things, Semantics, Cognitive Computing, and VANET.

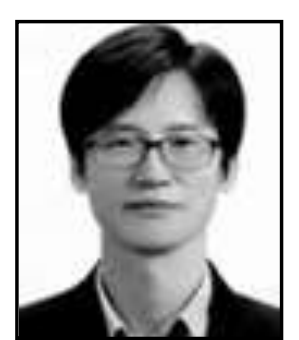

Si Ho Cha, he is a professor in the Department of Multimedia Science, Chungwoon University, Incheon, Korea. He received his $\mathrm{Ph} . \mathrm{D}$. degree in Computer Science from Kwangwoon University, Seoul, Korea in 2004. From 1997 to 2000, he worked as a senior researcher at Daewoo Telecom R\&D Center, Korea. His research interests include network management, wireless sensor networks, vehicular ad hoc networks, semantic web, and web of things. 
International Journal of Control and Automation

Vol. 11, No. 1 (2018) 